\title{
A functional polyvinyl alcohol fibrous membrane loaded with artemisinin and chloroquine phosphate
}

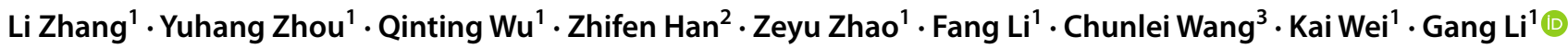

Received: 15 November 2020 / Accepted: 24 May 2021 / Published online: 31 May 2021

(c) The Polymer Society, Taipei 2021

\begin{abstract}
In order to reduce the infection rate of major respiratory diseases, it is crucial to development of masks with antibacterial functions. This paper describes an electrospun polyvinyl alcohol (PVA) fibrous membranes loaded with antibacterial drugs. The concentration of artemisinin (ART)/chloroquine phosphate (CQP) in PVA solution and the process parameters were investigated. Meanwhile, the physical properties and antibacterial effect, filtration performance, respiratory resistance and the surface wettability of drug membranes were tested and analyzed. The results showed that when the mass concentration of PVA was 9 wt. $\%$ and the dosage of ART/CQP was $1.5 / 10 \mathrm{mg} / \mathrm{mL}$, the fibers had uniform thickness and no beads. The prepared PVA fiber membrane has good hydrophilic, filtration and antibacterial properties, and the inhibition zone to Staphylococcus aureus and Escherichia coli was $7 \pm 0.3 \mathrm{~mm}$ and $4 \pm 0.2 \mathrm{~mm}$, respectively. The development of antibacterial PVA drug loaded fibrous membranes has potential value in the application of medical protective mask materials.
\end{abstract}

Keywords Antibacterial function $\cdot$ Electrospinning $\cdot$ Membrane $\cdot$ Artemisinin $\cdot$ Chloroquine phosphate

\section{Introduction}

In recent years, the severe respiratory infectious diseases such as severe acute respiratory syndrome (SARS), avian influenza (H1N1) and Ebola hemorrhagic fever (EBOV) have a high infection rate, especially the new coronavirus (COVID-19) that broke out the last year has brought serious impacts and threats to people's health and safety [1]. Masks

Li Zhang and Yuhang Zhou contributed to this manuscript equally

Zhifen Han

hzf_251014@163.com

$\triangle$ Kai Wei

weikai@suda.edu.cn

$\bowtie$ Gang Li

tcligang@suda.edu.cn

1 National Engineering Laboratory for Modern Silk, College of Textile and Clothing Engineering, Soochow University, Suzhou 215123, China

2 Department of Medical Oncology, Shuguang Hospital, Shanghai University of Traditional Chinese Medicine, Shanghai 201203, China

3 Sunna Technologies (Shanghai) Co. Ltd, Shanghai 201203, China are the simplest and most effective tool to resist respiratory infection. As early as 2007, the US Centers for Disease Control and Prevention had published interim guidelines, strongly recommending that people wear masks during an influenza outbreak [2]. However, most of the commercial masks only play a physical isolation role and do not have antibacterial functions. It brings another safety obstacle to the consumer, and also increases the difficulty of disposing of discarded masks. Therefore, it is of great significance to develop a mask with good antibacterial functions.

Currently, there are three main methods for antibacterial treatment of pharmaceutical protective masks [3, 4]: the first one is silver ions coated mask by using silver ions as antibacterial agents, which has strong antibacterial properties, but oxidation reduction reactions of silver ion antibacterial agents are prone to reduce antibacterial performance during the preparation process, and excessive silver ions may cause damage to the human body. The second one is drug coated mask by impregnating or coating with various drugs to make it antibacterial, but the drug loading is low, and the antibacterial time and effect are still insufficient due to the poor adhesion of masks and drugs. The third one is antibacterial materials woven mask made from antibacterial fibers such as chitin, chitosan, which can maintain antibacterial stability [4]. However, the traditional non-woven filter cloth 
has poor filtration performance for particles or bacteria with a size of submicron and nanometers [5]. Therefore, it is very important to develop masks with good filtration performance and antibacterial properties.

Polyvinyl alcohol (PVA) is a widely used medicinal polymer material due to its non-toxic to cells and the human body, and a nano-level PVA fiber with ultra-high filtration efficiency for tiny particles [6] can be spun, and the price is low, which is conducive to popularization and application. Although electrospinning nanofibers can filter particulates, they cannot inhibit or kill bacteria and viruses in the air. This is an urgent problem to be solved that the nanofiber membrane maintains high-efficiency filtration while achieving effective killing or inhibiting of bacteria and viruses [7].

Artemisinin (ART) is an active ingredient of anti-malarial extracted from the plant Artemisia annua, which has characteristics of antibacterial [8], antiparasitic, antipyretic, promoting immunity, low toxicity and no side effects [9] due to a variety of medicinal ingredients in it. Jiang et al. studied the antibacterial activity of artemisinin and its derivatives, the results showed that artemisinin and its derivatives had direct or synergistic antibacterial effects on Mycobacterium tuberculosis, Escherichia coli, Staphylococcus aureus and Helicobacter pylori [10]. Liu et al. investigated the effect of ART and its derivatives on the treatment of new coronavirus pneumonia, the results showed that artemisinin and its derivatives had definite anti-inflammatory and immunomodulatory effects, which can reduce the level of many inflammatory factors and inhibit the occurrence and development of new coronavirus (COVID-19) induced chronic subclinical systemic inflammation (CSSI). Its antibacterial effect can also inhibit bacterial infection [11]. Chloroquine phosphate (CQP) is an anti-malarial drug and can also be used for immune diseases such as parenteral amoebiasis, rheumatoid arthritis and nephrotic syndrome [12]. CQP can inhibit the replication of many diseases, such as influenza A [13], Zika virus [14], Ebola virus [15], SARS coronavirus [16] and Middle East syndrome coronavirus [17], etc. The latest research showed that CQP can effectively inhibit the activity of COVID-19 at the cellular level [1], and a number of clinical studies on CQP for the treatment of COVID-19 have been registered [18]. Mo et al. examined the clinical efficacy of CQP treatment in patients with COVID-19 after failure of Abidol treatment. The results showed that chloroquine phosphate treatment can be considered for patients with new coronary pneumonia that has failed the treatment of Arbidol, which has good clinical efficacy, mild adverse reactions, and can be relieved after stopping the drug [19].

In this paper, water-soluble PVA loaded with ART and CQP drugs were used to prepare drug loaded nanofiber membranes with antibacterial properties using electrospinning technology. The concentration of ART/CQP in PVA solution and the process parameters were investigated.
Meanwhile, the physical properties and antibacterial effect, filtration performance, respiratory resistance and the surface wettability of drug membranes were tested and analyzed.

\section{Materials and methods}

\section{Materials}

The following materials were prepared: PVA (type 1788, Wuxi Yatai United Chemical Co., Ltd, China); Artemisinin (ART, Shanghai Tichiai Chemical Industry Development Co., Ltd, China); Chloroquine phosphate (CQP, Nanjing Dulai Biotechnology Co., Ltd, China). All of the chemicals and reagents are analytical grade.

\section{Preparation of drug-loaded nanofibers}

PVA solution with concentration of $8-10 \mathrm{wt}$.\% was prepared, and then absolute ethanol dissolved ART and CQP were mixed with PVA solution until completely dissolved. The blended solution was injected into a syringe with a volume of $20 \mathrm{~mL}$ and a needle diameter of $1.0 \mathrm{~mm}$, which fixed on the micro-injection pump. The needle worked as the positive electrode and the electrospinning silicone oil paper worked as the negative electrode. The spinning voltage was $16-20 \mathrm{kV}$, and the environmental humidity was around $50 \%$ during the spinning process. The electrospinning silicone oil paper was fixed $15-20 \mathrm{~cm}$ far away from the needle on the drum to collect nanofibers.

\section{Microscopic observations}

The PVA drug-loaded nanofiber membrane was pasted on the electron microscope sample table with conductive glue before spraying gold on the sample for $90 \mathrm{~s}$, and then the sample was observed by using field emission scanning electron microscope (SEM, S4800, Hitachi, Japan) and desktop scanning electron microscope (TM, TM3030, Hitachi, Japan).

\section{Fourier infrared spectroscopy}

Nicolet 5700 Fourier Infrared Spectrometer (FTIR, Nicolet, USA) was used to demonstrate the shift of specific absorption peaks of $600-4000 \mathrm{~cm}^{-1}$ in the chromatogram of PVA drug-loaded nanofiber membranes with different compositions and ratios.

\section{Hydrophilic test}

The surface wettability (hydrophilicity) of the PVA drugloaded fiber membrane was measured with the SL200B 
contact angle tester. A $30 \mu \mathrm{L}$ deionized water drop was used to fell on the surface of the fiber, a high-speed camera was used to shoot, and the contact angle value was calculated by software. Each sample was tested for 3 times and the average value was calculated.

\section{Mechanical performance test}

An Instron 3365 material testing machine was used to test the mechanical properties of the fiber membrane. The fiber membrane was cut into a rectangle with a width of $1 \mathrm{~cm}$ and a length of $6 \mathrm{~cm}$, and the tensile speed was set to $10 \mathrm{~mm} /$ min for testing. Each sample was tested 3 times and the average value was taken.

\section{Antibacterial properties assessment}

Gram-positive Staphylococcus aureus and Gram-negative Escherichia coli were selected to prove the antibacterial activity of the PVA drug-loaded fiber membrane. Every sample was cut into a circle with the same shape, and inoculated approximately $1 \mathrm{~mL}$ of bacterial suspension $\left(1 \times 10^{8} \mathrm{CFU} /\right.$ $\mathrm{mL}$ ) on an agar culture plat in a $37^{\circ} \mathrm{C}$ incubator for $18-24 \mathrm{~h}$. According to the GB/T 20,944.1-2007, the antibacterial effect of the sample was judged by the size of its inhibition zone. The calculation formula of the inhibition zone is shown in formula (1):

$H=(D-d) / 2$

where $\mathrm{H}$ represents width of the inhibition zone $(\mathrm{mm}), \mathrm{D}$ represents the average value of the outside diameter of the inhibition zone $(\mathrm{mm}), \mathrm{d}$ represents sample diameter $(\mathrm{mm})$.

\section{Filtration performance measurement}

The drug-loaded PVA fiber membrane was cut into a size of $20 \times 20 \mathrm{~cm}$, and placed as an intermediate layer between the inner and outer layers of a standard medical mask. According to the standard GB/T 19,083-2010, the automatic filter tester 8130 of TSI Company of the United States was used to test the filtration efficiency and filtration resistance sample three times under the condition of a gas flow at $85 \mathrm{~L} / \mathrm{min}$ and $\mathrm{NaCl}$ aerosol particles with diameter at $0.06-0.08 \mu \mathrm{m}$.

\section{Surface water resistance and wetness determination}

Y813 fabric wetness tester was used to evaluate the wetness level of the sample. Installing the sample on the ring holder at $45^{\circ}$, and spraying $250 \mathrm{~mL}$ distilled water on the surface of fabric. According to the comparison wetness phenomenon between the appearance of the sample and the standard picture, the wet level and the waterproof performance of the sample were evaluated.

\section{Results and discussion}

\section{Comparison of different PVA drug-loaded fiber membranes}

The different parameters of PVA drug-loaded fibrous membranes were given in Table 1. The TM images of PVA drug-loaded membranes were shown in Fig. 1. The results showed that PVA concentration and drug loading dosage have important effects on the morphology of nanofibers. When the PVA concentration is $8 \mathrm{wt}$. \%, the viscosity of the solution is lower. In the electrostatic field, the solution is stretched by force, because of the viscoelastic effect between polymer molecular chains, the solution jet tends to shrink, causing the PVA solution to agglomerate and then form polymer beads. As the PVA concentration increases, the beads decreased and finally disappeared, and the fiber diameter becomes thicker, increasing from 257 to $458 \mathrm{~nm}$. In comparison, the electrospinning fibers which obtained from a, b, c and d group parameters given in Table 1 have uniform fineness, and the fibers are continuous and smooth. Hence, this optimized technical parameter can be used for the subsequent preparation of nanofiber membranes.

In this work, drug-loaded nanofiber membrane was prepared and used as the middle layer material of the mask. Firstly, the non-woven fabric was used as the receiver directly, and then the PVA drug-loaded fiber membrane was sprayed onto the non-woven fabric using electrospinning technology. The groups a, b, c and d obtained from the above experiments were selected for further characterization. In order to control the respiratory resistance of mask, the spinning time for the drug-loaded fiber membrane was controlled at $2 \mathrm{~h}$, and the bolus injected speed was maintained at $0.3 \mathrm{~mL} / \mathrm{h}$. The SEM images of the membrane-coated non-woven fabric a, b, c and d obtained by optimized electrospinning parameters were shown in Fig. 2. Due to the control of the spinning time and the injection speed, the space between the fibers is uniform, and the diameter distribution of the fiber is between $100-700 \mathrm{~nm}$. According to the statistics of diameter distribution, the thickness of the electrospinning fibers in group c is uniform and its diameters are around $200-400 \mathrm{~nm}$. The experimental results showed that the electrospinning fibers can be stably covered on the surface of the non-woven fabric, and the fiber is smooth and uniform. 

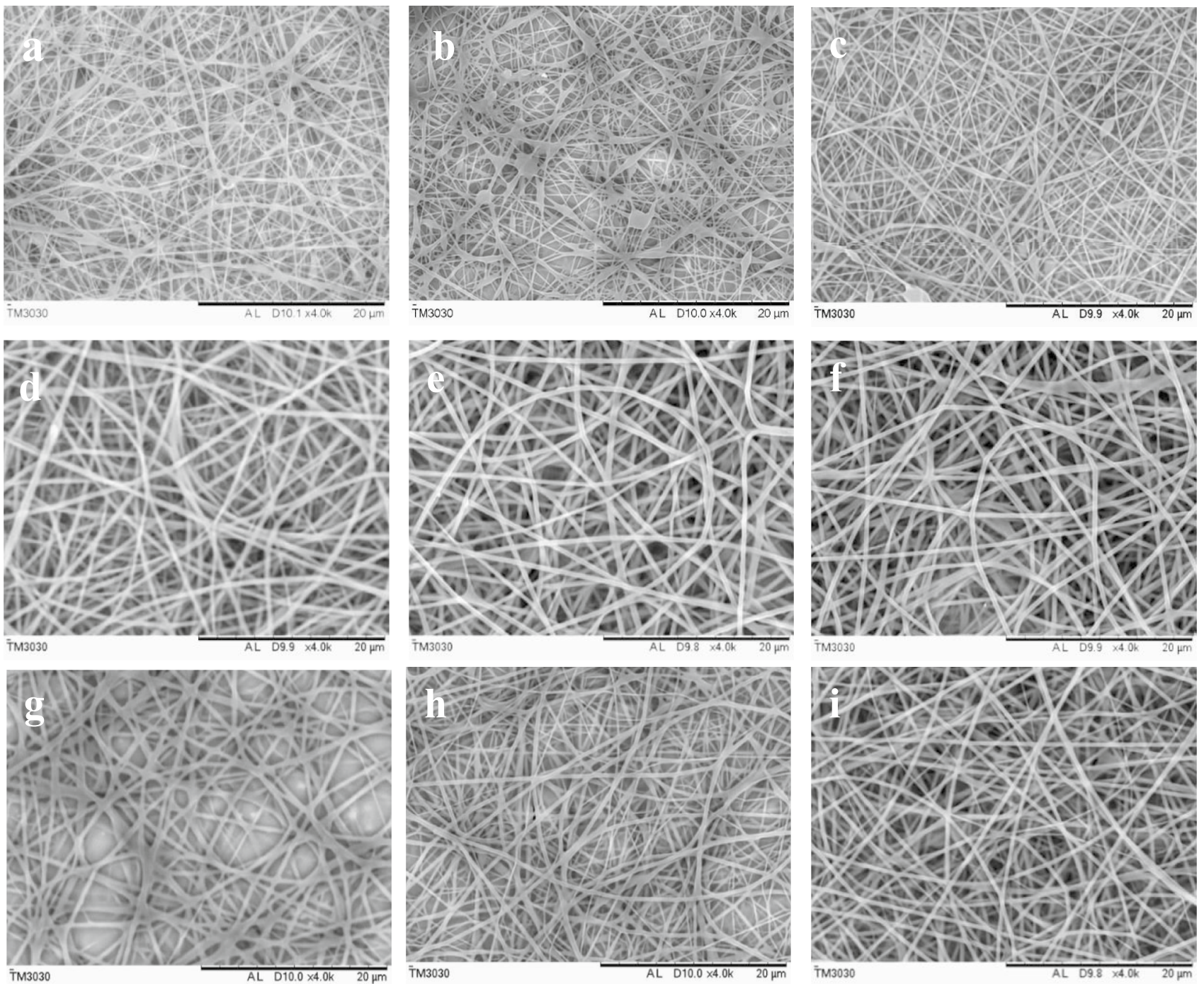

Fig. 1 TM images of PVA drug-loaded fibrous membranes with different processing parameters: (a-i) represent different samples (Table 1)

\section{FT-IR analysis}

The changes on the specific chemical groups and structure of the drug-loaded PVA fibrous membrane were determined by infrared spectrum. The infrared spectrum results of the drug-loaded PVA fiber membranes ( $a, b, c$, and d) were shown in Fig. 3. The characteristic absorption peaks of PVA can be obtained: $3288 \mathrm{~cm}^{-1}$ is the stretching vibration of the carboxyl group, $2918 \mathrm{~cm}^{-1}$ is the stretching vibration peak of the methylene group, and $837 \mathrm{~cm}^{-1}$ is the stretching vibration peak of the $\mathrm{C}=\mathrm{C}$ bond. The special absorption peaks of ART are: the stretching vibration peak of the carbonyl group at $1738 \mathrm{~cm}^{-1}$ and the stretching vibration peak of the carbon-oxygen group at $1116 \mathrm{~cm}^{-1}$ and the stretching vibration peak of $\mathrm{Cl}^{-1}$ in CQP at $615 \mathrm{~cm}^{-1}$. It can be seen that electrospinning process did not change the structure of ART and CQP.
Hence, the both drugs were successfully loaded into the PVA fibrous membranes.

\section{Mechanical properties of drug-loaded fiber membranes}

As the middle layer of the mask, it not only has good filtration performance, moisture absorption and air permeability but also has remarkable mechanical properties. During the wearing process, the shape of the mask is constantly changing, so the material should also have excellent elasticity. As shown in Fig. 4, the PVA concentration in the groups a, b, and c was 9 wt. \%, the breaking strength and elongation at break did not change much, the breaking strength was among $29-35 \mathrm{cN}$, and the elongation at break was among $75 \%-100 \%$. The concentration of PVA in group d increased to $10 \mathrm{wt}$ \%, 



Fig. 2 SEM images of electrospinning PVA fibrous membranes loaded with different content of ART/CQP: a, b, $\mathbf{c}$ and $\mathbf{d}$ represents membranes of PVA, ART/CQP at 9 wt. $\%, 0.5 / 50 \mathrm{mg} / \mathrm{mL} ; 9$ wt. $\%, 1 / 25 \mathrm{mg} / \mathrm{mL} ; 9$ wt. $\%, 1.5 / 10 \mathrm{mg} / \mathrm{mL}$ and $10 \mathrm{wt} . \%, 1.5 / 10 \mathrm{mg} / \mathrm{mL}$, respectively

and its drug loading was the same as group c, but the breaking strength and elongation at break both increased significantly. Specifically, the breaking strength increased from 25 to $37 \mathrm{cN}$, and elongation at break increased from $83 \%$ to $140 \%$. This is because the increase of concentration of PVA makes the fiber diameter thicker, and the fibers are tightly bonded and are hardly to slip, inducing the increase of mechanical strength, which is consistent with the statistical results of the fiber diameter given in Table 1.

\section{Hydrophilic property of drug-loaded fiber membranes}

The outer layer of the mask usually has good hydrophobic performance, while the inner layer is a skin-friendly layer, which should have good comfort properties. During the use of the mask, liquid droplets are generated due to the gas exchange between the inside and outside of the mask. In order to maintain good moisture absorption and 


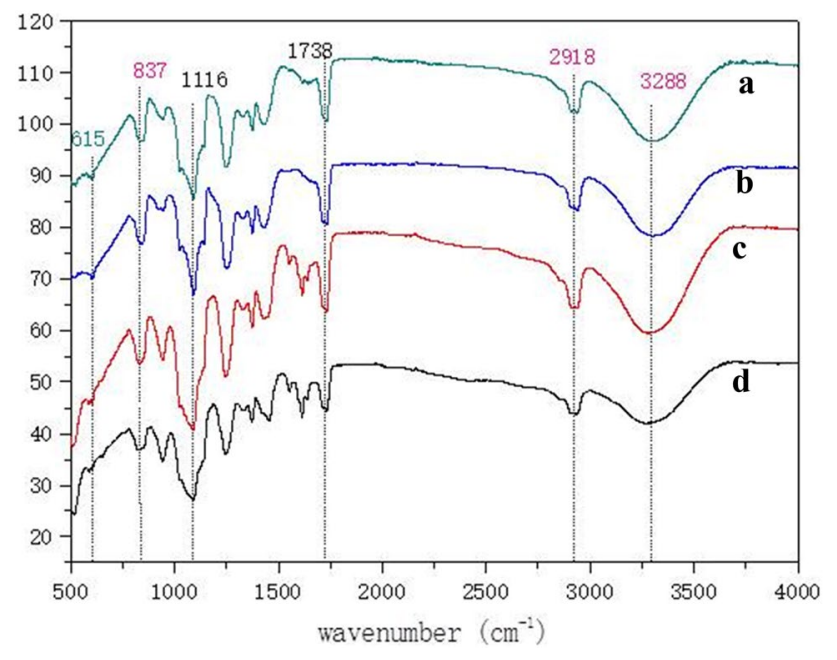

Fig. 3 FT-IR images of electrospinning PVA fibrous membranes loaded with different content of ART/CQP: (a-d) represent different samples (Table 1)

air permeability of the mask, the middle layer needs to have good hydrophilicity property. The hydrophilic test results of the fiber membrane in 0-20 s were shown in Fig. 5a. As time proceeds, the water droplets become smaller. When it is stable at $20 \mathrm{~s}$, only a small amount of water droplets stay on the surface of the fibrous membrane. This is because the existence of hydroxyl groups in PVA that makes PVA has better hydrophilic property. The statistical analysis results of the water contact angle were shown in Fig. 5b. The results of surface hydrophilic effect of the PVA drug-loaded fibrous membrane is consistent with the images of contact angle, the water contact angle is between $20-40^{\circ}$. Upon the concentration of PVA remains the same, the contact angle decreases with the increase of the CQP loading dose. This is because the CQP is easily soluble in water and can absorb part of the water after contacting with water, which increases the hydrophilic effect of the fibrous membrane. By comparing groups $\mathrm{c}$ and $\mathrm{d}$ with the same drug loading dose, it is found that due to the presence of hydrophilic groups in PVA, the higher the PVA concentration, the better the hydrophilic performance of the fibrous membrane.

\section{Filtering performance and breathing resistance of mask}

The filtering effect is an important property for evaluating the quality of masks. The results of the filtration performance test of the PVA drug-loaded fibrous membrane as the middle layer of the mask were shown in Fig. 6. The bolus injection speed was controlled at $0.3 \mathrm{~mL} / \mathrm{h}$, the spinning time was $2 \mathrm{~h}$, and the corresponding gram weight of the PVA fibrous membrane is $2 \mathrm{~g} / \mathrm{m}^{2}$. The fibrous membranes with a gram weight of $2 \mathrm{~g} / \mathrm{m}^{2}$ were placed as an intermediate layer between the inner and outer layers of a commercial medical mask to test the total filtration performance. During the testing process, the gas flow rate was $85 \mathrm{~L} / \mathrm{min}$ and the $\mathrm{NaCl}$ aerosol particles were $0.07 \mu \mathrm{m}$. The results were shown in Fig. 6a: when the gram weight is $2 \mathrm{~g} / \mathrm{m}^{2}$, the filtration efficiency is $80-87 \%$, and the filtration resistance is 799-1050 Pa.

A good filtration performance of the mask is an important indicator to evaluate its performance. In addition, there are
Fig. 4 Mechanical property characterization of electrospinning PVA fibrous membranes loaded with different content of ART/CQP: (a-d) represent different samples (Table 1)

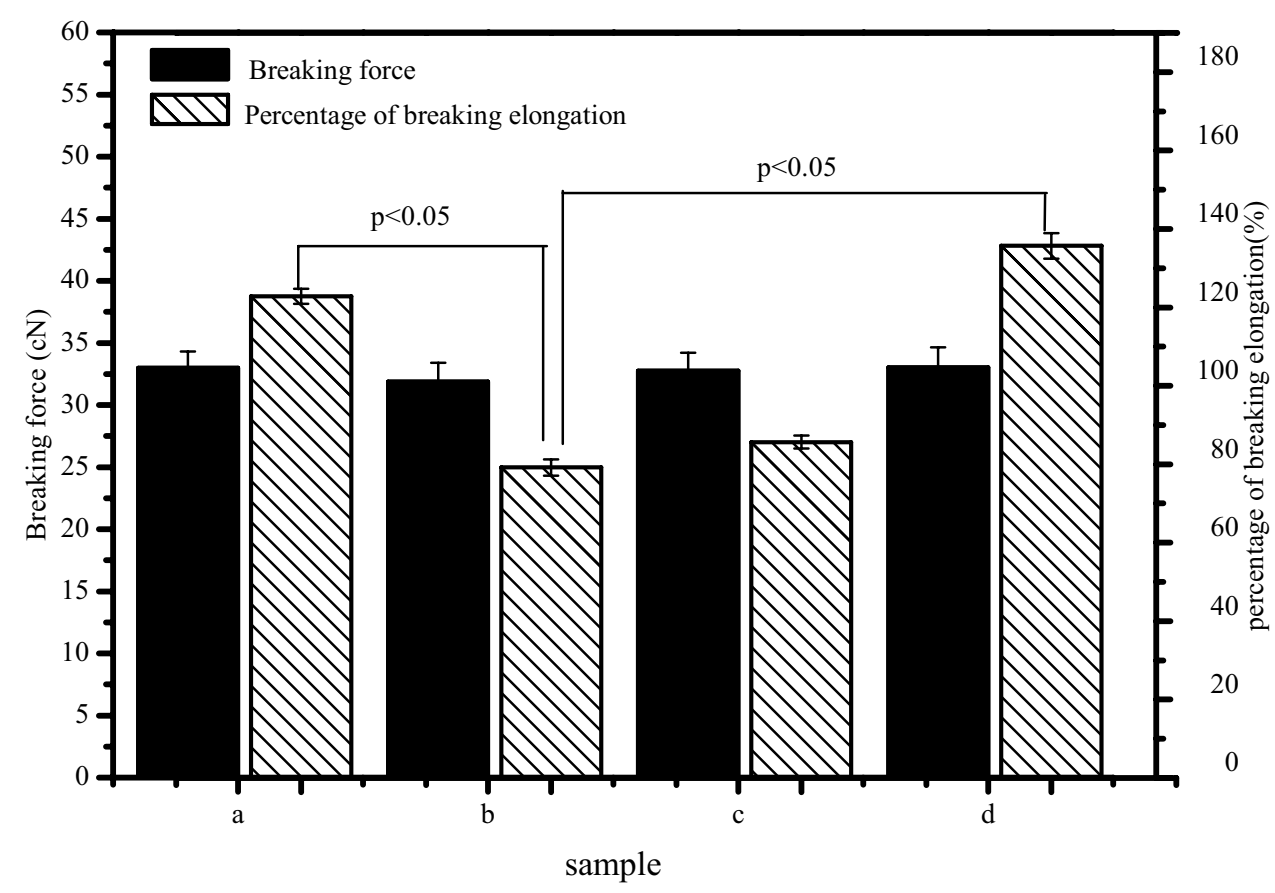


Fig. 5 Images of water contact angle (a) and quantitatively analysis (b) of electrospinning PVA fibrous membranes loaded with different content of ART/ CQP: (a-d) represent different samples (Table 1)

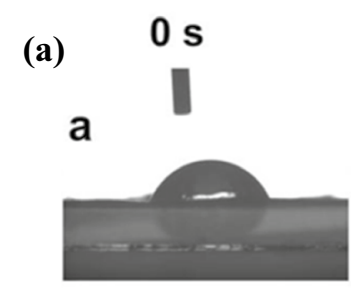

b
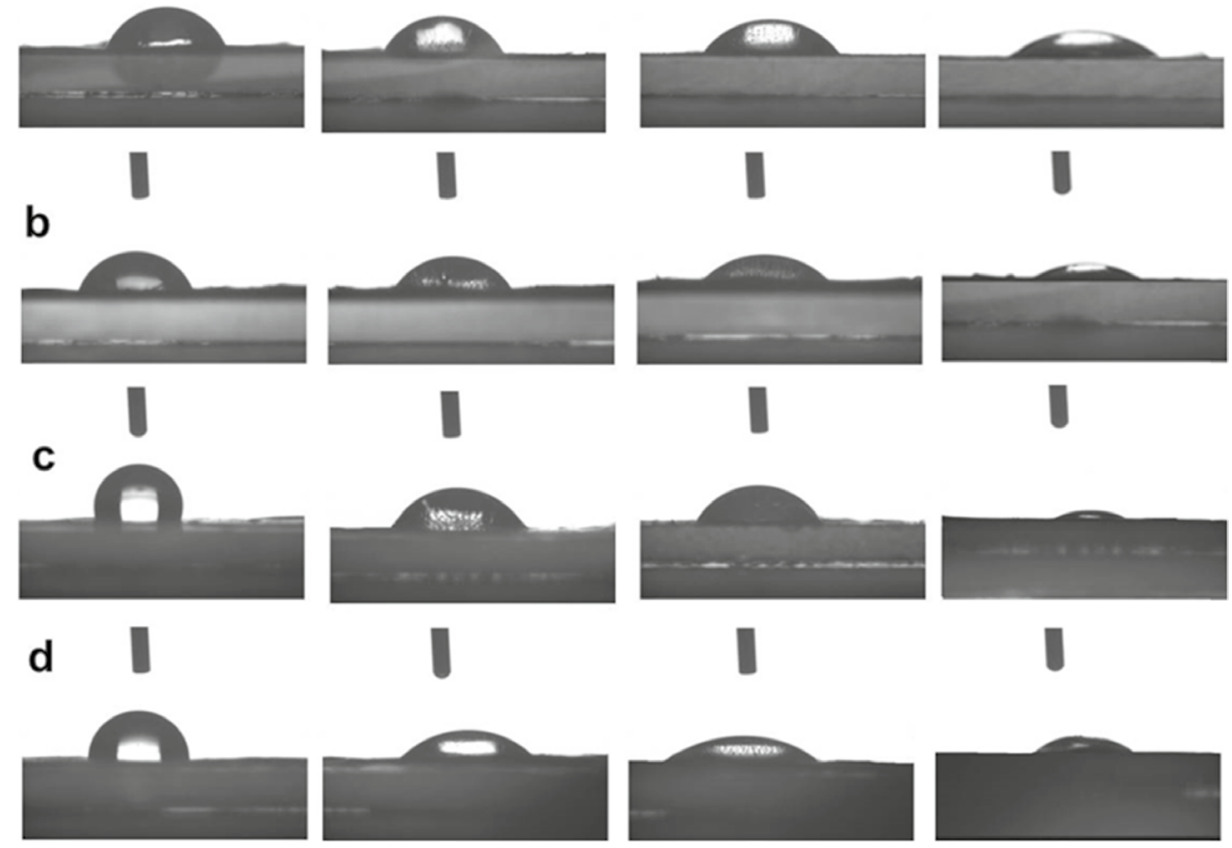

】
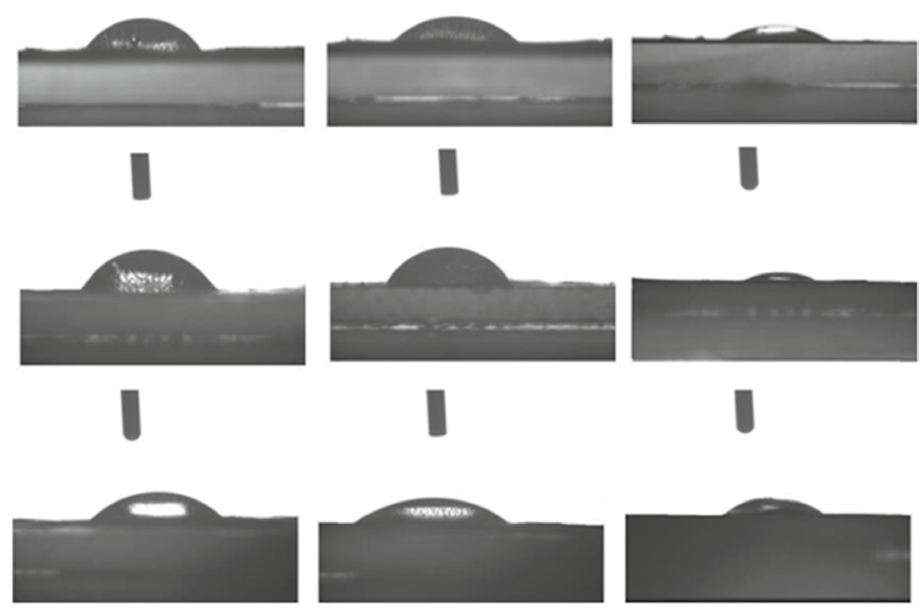

(b)

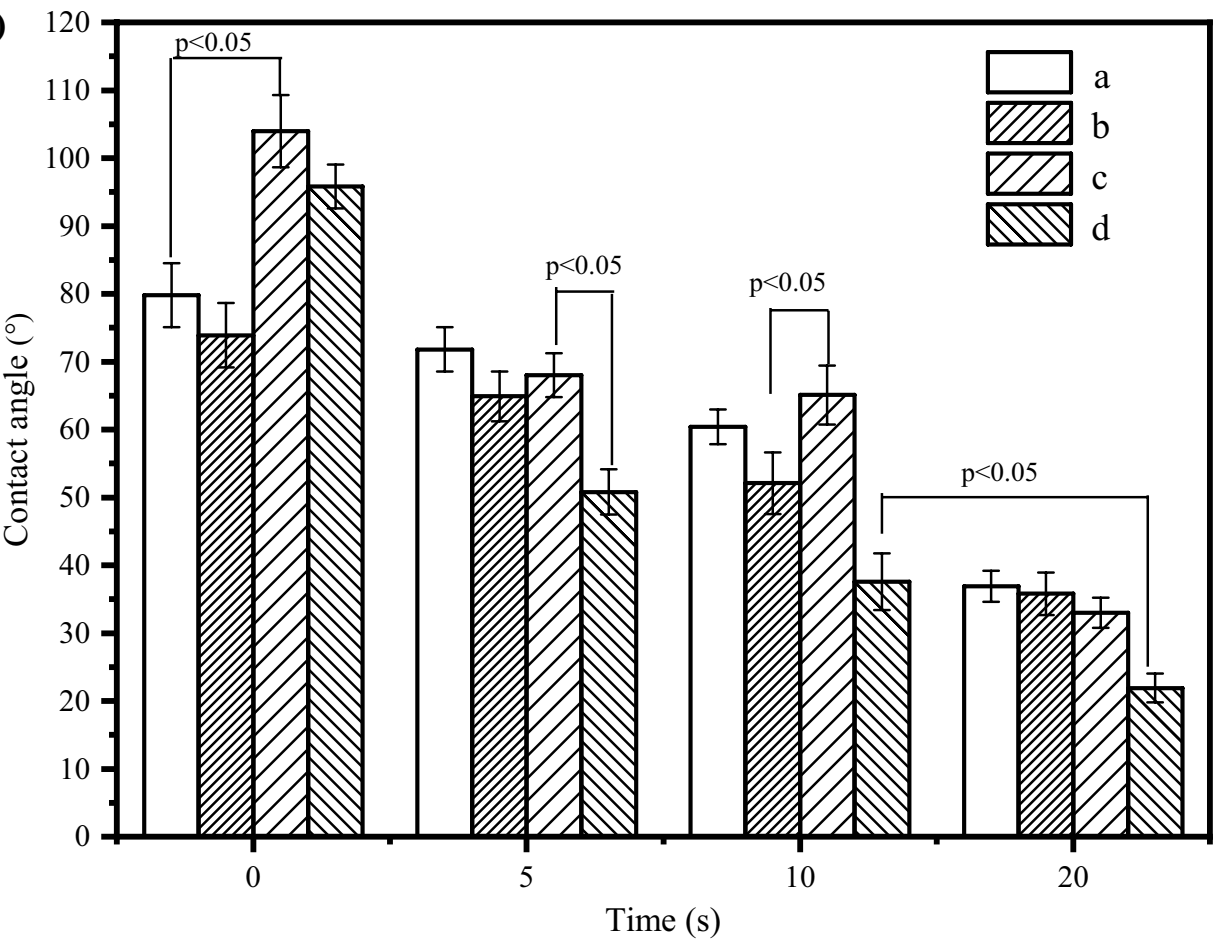

also certain requirements for the respiratory resistance of masks. Usually, for the same gram-weight, fiber diameter and number, pore size and distribution of fibrous membrane, the relationship between the breathing resistance and the filtration efficiency is positive, namely, the breathing resistance increases with the increase of the filtration efficiency. But, the high breathing resistance induces uncomfortable wearing feeling to peoples, while the low breathing resistance causes low filtration efficiency and so as to high risk for peoples. So it is very important to find an optimal balance relationship between filtration efficiency and breathing resistance. The respiratory resistance test was carried out on the fibrous 
Fig. 6 Filtration performance of PVA fibrous membranes loaded with different amounts of ART/CQP; (a-d) represent different samples (as described in Table 1); (a) filtration efficiency; (b) filtration resistance

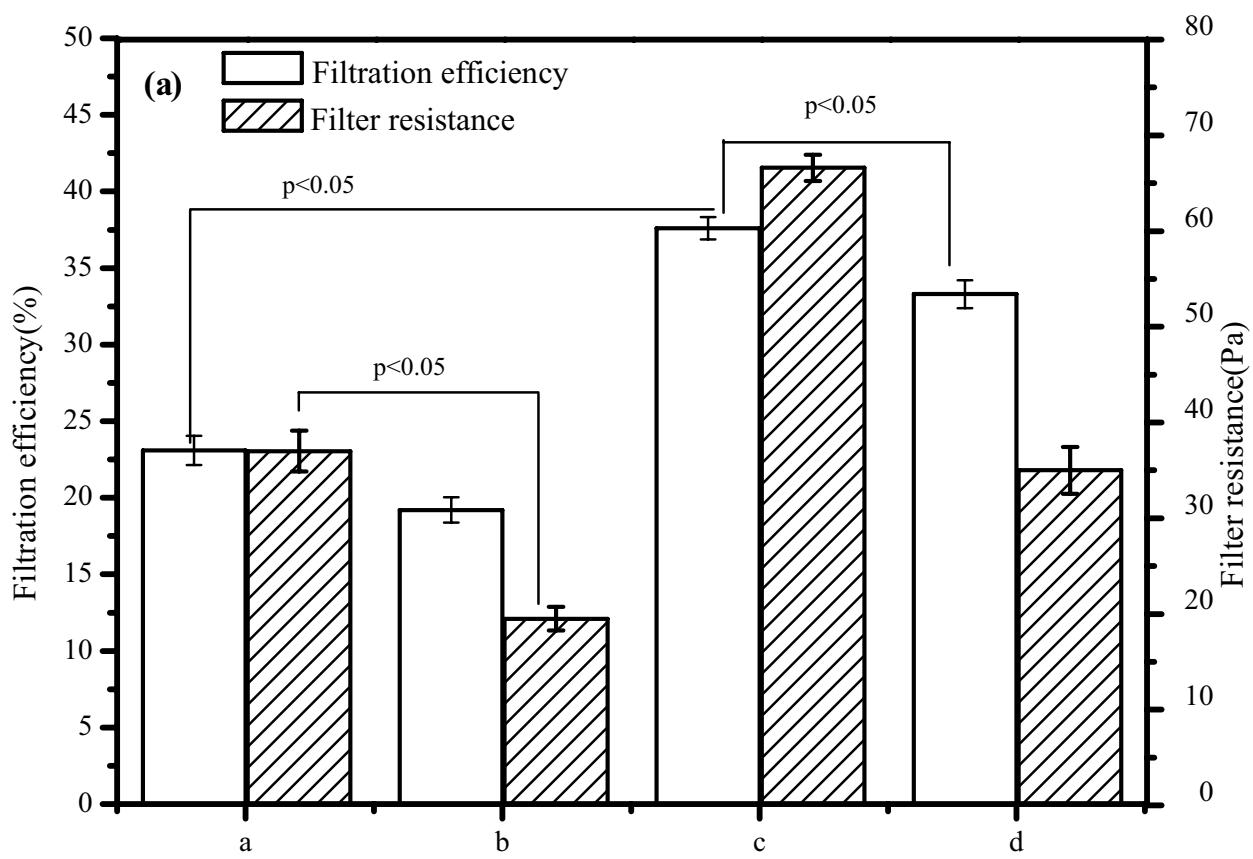

Sample

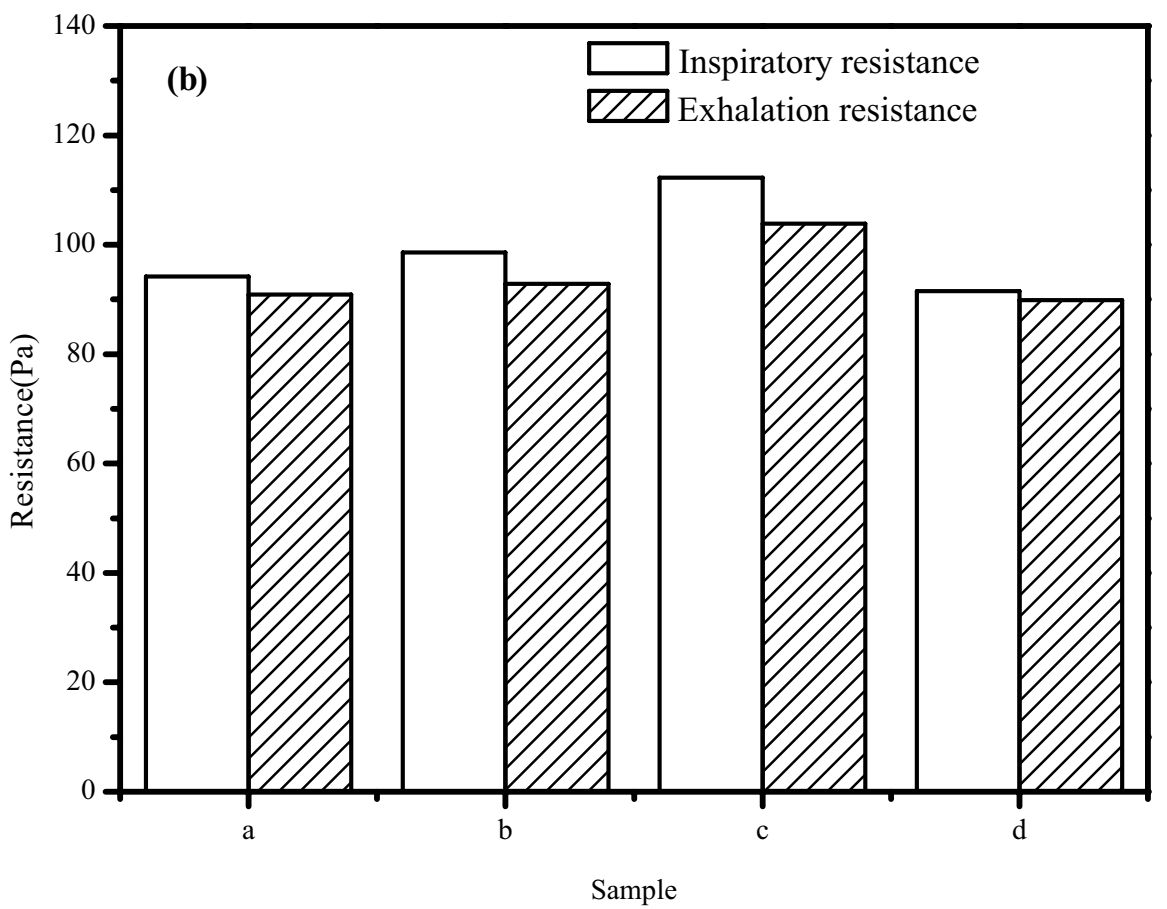

membrane with a gram weight of $2 \mathrm{~g} / \mathrm{m}^{2}$. The respiratory resistance of the sample is 90-110 Pa. As shown in Fig. 6b, when the PVA concentration keeps the same, as the drug loading decreases, the fiber diameter increases as well as its distribution becomes more uniform, while the space between fibers decrease, inducing the increase of filtration efficiency and respiratory resistance. With the increase of PVA concentration, the fiber diameter increases, inducing the increase of the filtration performance and respiratory resistance. Hence, it is a balance to control the high respiratory resistance on the basis of ensuring the filtration efficiency.

\section{Waterproofing and wetting grade of the surface}

As shown in Fig. 7, according to the standard of waterwetting grade in GB/T4745-2012, it can be seen that the surface of the drug-loaded fibrous membrane is completely wetted, and the water-wetting grade stands for grade 1 , 
Fig. 7 Wettability images of electrospinning PVA fibrous membranes loaded with different content of ART/CQP: (a-d) represents membranes of PVA and ART/CQP at 9 wt. $\%$ \& $0.5 / 50 \mathrm{mg} / \mathrm{mL} ; 9$ wt. $\%$ \& $1 / 25 \mathrm{mg} / \mathrm{mL} ; 9$ wt. $\%$ \& $1.5 / 10 \mathrm{mg} / \mathrm{mL}$ and $10 \mathrm{wt} . \%$ \& $1.5 / 10 \mathrm{mg} / \mathrm{mL}$, respectively
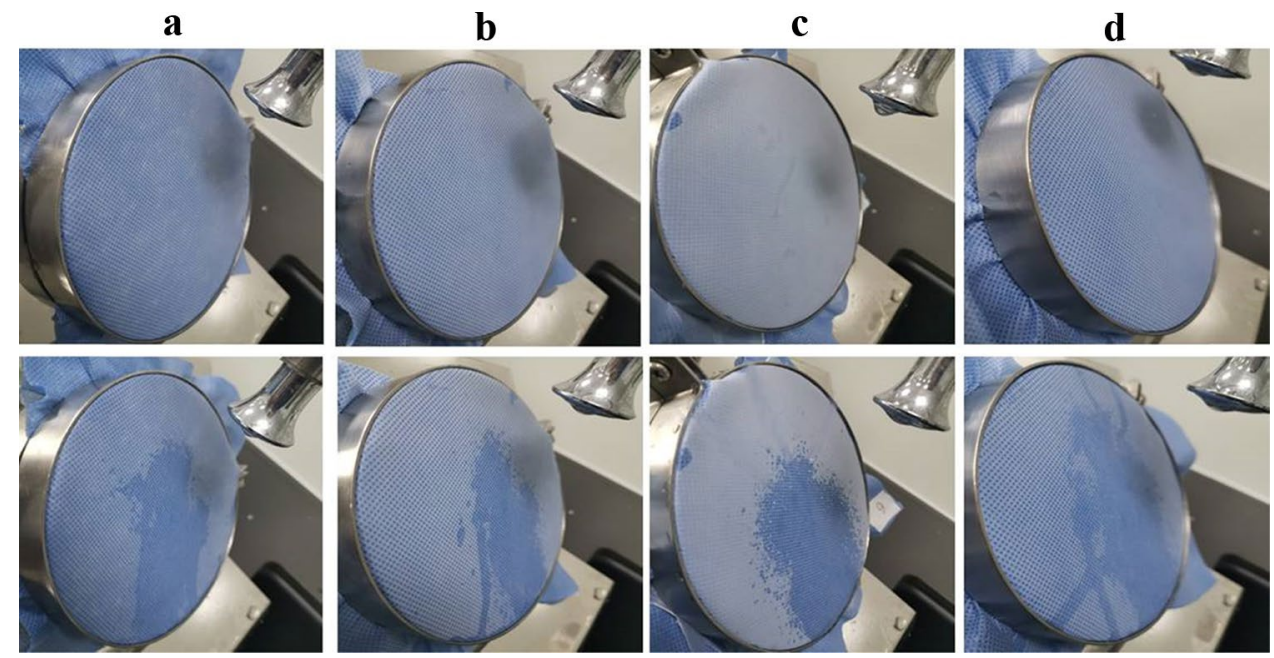

indicating poor waterproof-performance, the results is consistent with hydrophilic result. In view of the hydrophobic medical non-woven fabric is used on the inner and outer sides of the mask, so the overall water-wetting effect of the mask is not greatly affected. A certain water-wetting property of the middle layer can increase the wearing comfort of the mask in use and keep the internal environment of the mask in dry condition.

\section{Antibacterial properties of drug-loaded PVA fibrous membrane}

Various bacteria carried by the human body can be exchanged with the external environment during the breath. Compared with the outside environment of the mask, the inside environment of the mask is more likely to produce bacteria, so it is crucial for the mask possessing antibacterial properties [5, 19]. As shown in Fig. 8, images a-d are the antibacterial results of the samples against Staphylococcus aureus (S.aureus) and Escherichia coli (E.coil). As the fibrous membrane contains ART with antibacterial effect, the fibrous membrane has good antibacterial effect [20]. The width of the antibacterial zone of S.aureus is $2 \pm 0.3 \mathrm{~mm}$, $3 \pm 0.4 \mathrm{~mm}, 7 \pm 0.3 \mathrm{~mm}$ and $3 \pm 0.2 \mathrm{~mm}$, respectively. The width of the antibacterial zone of E.coli is $2 \pm 0.1 \mathrm{~mm}$, $3 \pm 0.2 \mathrm{~mm}, 4 \pm 0.2 \mathrm{~mm}$ and $2 \pm 0.1 \mathrm{~mm}$, respectively, according to the GB/T20944.1-2007 standard of textile antibacterial performance, the antibacterial zone of the samples is more than $1 \mathrm{~mm}$, showing a good antibacterial effect and antibacterial performance. Among them, group $\mathrm{c}$ has the best antibacterial effect. This is because that the antibacterial effect was significantly improved with the increase of ART content in membranes.
Fig. 8 Antibacterial images of electrospinning PVA fibrous membranes loaded with different content of ART/CQP: (a-d) represents membranes of PVA and ART/CQP at 9 wt. $\%$ \& $0.5 / 50 \mathrm{mg} / \mathrm{mL} ; 9$ wt. $\%$ \& $1 / 25 \mathrm{mg} / \mathrm{mL} ; 9$ wt. $\%$ \& $1.5 / 10 \mathrm{mg} / \mathrm{mL}$ and $10 \mathrm{wt} . \% \&$ $1.5 / 10 \mathrm{mg} / \mathrm{mL}$, respectively a
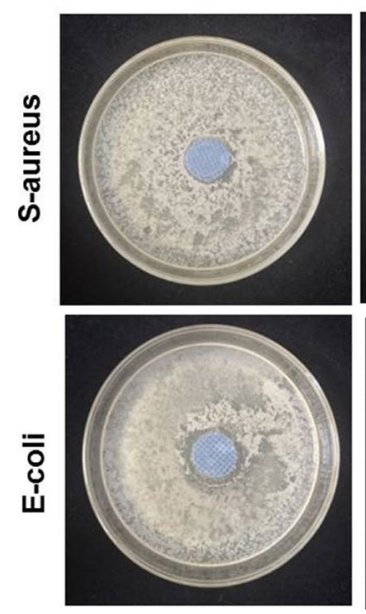

b
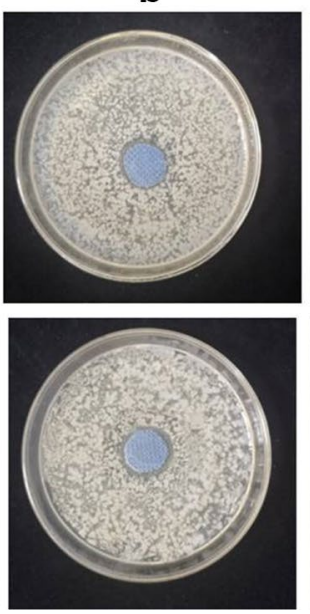

C
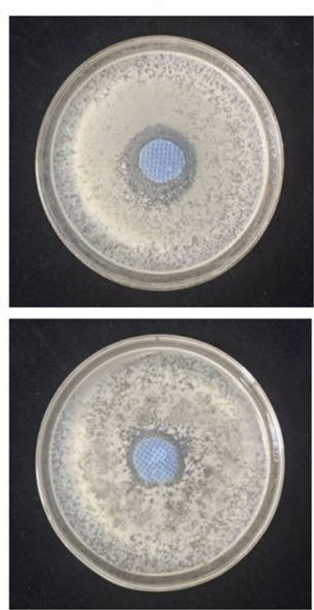

d
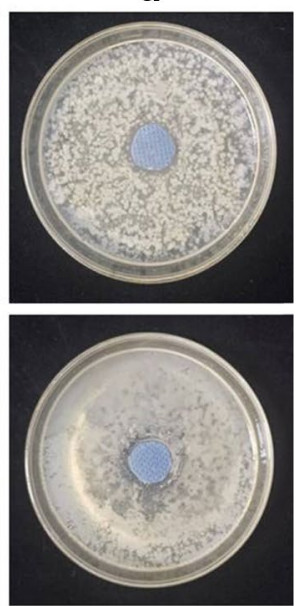
Tab. 1 Processing parameters of PVA drug-loaded nanofiber membranes

\begin{tabular}{llllllll}
\hline Item & $\begin{array}{l}\text { PVA } \\
(\mathrm{wt} \%)\end{array}$ & $\begin{array}{l}\text { ART } \\
(\mathrm{mg} / \mathrm{mL})\end{array}$ & $\begin{array}{l}\text { CQP } \\
(\mathrm{mg} / \mathrm{mL})\end{array}$ & $\begin{array}{l}\text { Spinning dis- } \\
\text { tance }(\mathrm{cm})\end{array}$ & $\begin{array}{l}\text { Voltage } \\
(\mathrm{kV})\end{array}$ & $\begin{array}{l}\text { Environmental } \\
\text { humidity }(\%)\end{array}$ & Diameter (nm) \\
\hline $\mathrm{a}$ & 9 & 0.5 & 50 & 15 & 20 & 45 & $371 \pm 6$ \\
$\mathrm{~b}$ & 9 & 1.0 & 25 & 15 & 20 & 45 & $377 \pm 4$ \\
$\mathrm{c}$ & 9 & 1.5 & 10 & 15 & 20 & 45 & $376 \pm 3$ \\
$\mathrm{~d}$ & 10 & 1.5 & 10 & 15 & 20 & 45 & $383 \pm 7$ \\
$\mathrm{e}$ & 8 & 0.5 & 50 & 15 & 20 & 45 & $283 \pm 5$ \\
$\mathrm{f}$ & 8 & 1.0 & 25 & 15 & 20 & 45 & $257 \pm 11$ \\
$\mathrm{~g}$ & 8 & 1.5 & 10 & 15 & 20 & 45 & $270 \pm 7$ \\
$\mathrm{~h}$ & 10 & 0.5 & 50 & 15 & 20 & 45 & $247 \pm 5$ \\
$\mathrm{i}$ & 10 & 1.0 & 25 & 15 & 20 & 45 & 247 \\
\hline
\end{tabular}

6. Shen B, Zhang D, Wei Y, Zhao Z, Ma X (2019) Preparation of Ag doped keratin/PA6 nanofiber membrane with enhanced air filtration and antimicrobial properties. Polym 11(9):1511-1524

In this work, we developed PVA-based fibrous membranes loaded with ART/CQP using electrospinning technique. The diameter and thickness of PVA drug-loaded fibrous membrane prepared under the optimized process parameters were uniform, and the surface was compact and smooth. The electrospinning process has little impact on the drug structure, and the fibrous membrane has good elasticity and hydrophilic properties. With the addition of ART/ CQP, the membrane has a certain antibacterial effect on Staphylococcus aureus and Escherichia coli, besides, the antibacterial effect was enhanced with the increase of ART loading dose. And when the membrane was used as the middle layer of the mask, the respiratory resistance is high, but the filtration efficiency was much higher than that of the commercial mask. The development of antibacterial PVA drug loaded fibrous membranes has potential value in the application of medical protective mask materials.

Acknowledgements This work was supported by National Key R\&D Program of China (2019YFE0117700), State Key Laboratory for Modification of Chemical Fibers and Polymer Materials-Donghua University (YJ202003) and "Top six talent peaks" program of Jiangsu (GDZB035). The authors would like to thank for the support of China National Textile and Apparel Council (J202002).

\section{References}

1. Wang M, Cao R, Zhang L, Yang X, Xiao G (2020) Remdesivir and chloroquine effectively inhibit the recently emerged novel coronavirus (2019-nCoV) in vitro. Cell Res 30:269-271

2. Mitka M (2007) Face masks, respirators might help during pandemic flu outbreak. JAMA J Am Med Assoc 297:2338

3. Li H, Song M, Wu J, Tian T, Qi J, Yang J (2011) Research progress of biological protective mask materials and protective performance. China Public Health 27:634-637

4. Liu Z, Zhao J, Li W, Li Y (2016) Computational screen and experimental validation of anti-influenza effects of quercetin and chlorogenic acid from traditional Chinese medicine. China J Pathogen Biol 6:19095

5. Xie X, Yu J, Zhao Z, Zheng Z, Li G (2019) Fabrication and drug release properties of curcumin-loaded silk fibroin nanofibrous membranes. Adsorpt Sci Technol 37:5-6
7. Yuan J, Wang S, Cui X (2009) Study on chemical composition and antibacterial activity of volatile oil from zanthoxylum bungeagum maxim. Prog Mod Biomed 34(10):1690-1697

8. Yan S, Yu J, Wang Y, Cai W, Weng X (2019) Research progress of effect of artemisinin family drugs on $\mathrm{T}$ lymphocytes immunomodulation. China J Chin Mater Med 44(22):4992-4999

9. Jiang W, Qian Y (2019) Research progress on antibacterial activity of artemisinin and its derivatives. Chin J Primary Med Pharm 30(014):2003-2007

10. Liu G, Cai N, Xie J, Xin C, Li R, Zhou H (2019) Discussion on the use of artemisinin and its derivatives in the treatment of novel coronavirus pneumonia. Drug Eval Res 43(004):606-612

11. Domenico P, Tatiana K (2018) Current and future use of chloroquine and hydroxychloroquine in infectious, immune, neoplastic, and neurological diseases: a mini-review. Clin Drug Invest 38:653-671

12. Ooi E, Chew J, Loh J, Chua RC (2006) In vitro inhibition of human influenza A virus replication by chloroquine. J Virol J 3:39

13. Delvecchio R, Higal M, Pezzutop P, Ana V, Patricia G, Fabio M, Erick L, Fabio $S$ (2016) Chloroquine, an endocytosis blocking agent, inhibits zika virus infection in different cell models. Viruses 8:322

14. Falzarano D, Safronet D, Prescott J, Marzi A, Feldmann F, Feldmann $\mathrm{H}$ (2015) Lack of protection against ebola virus from chloroquine in mice and hamsters. Emerging Infect Dis 21:1065-1067

15. Vincent MJ, Bergeron E, Benjannet S, Erickson BR, Nichol ST (2005) Chloroquine is a potent inhibitor of SARS coronavirus infection and spread. Virol $\mathrm{J}$ 2:69-72

16. Dyall J, Coleman CM, Hart BJ, Venkataraman T, Holbrook MR, Kindrachuk J, Johnson RF (2014) Repurposing of clinically developed drugs for treatment of middle east respiratory syndrome coronavirus infection. Antimicrob Agents Chemother 58:4885-4893

17. Andrea C, Giulia I, Mariachiara I (2020) A systematic review on the efficacy and safety of chloroquine for the treatment of COVID-19. J Crit Care 57:279-283

18. Mo G, Xi J (2020) Observation on the efficacy of chloroquine phosphate in the treatment of patients with new coronary pneumonia who failed Abidol treatment. Adver Drug React J 5:25-29

19. Tara O, Brosseau LM (2008) Surgical mask filter and fit performance. Am J Infect Control 3:276-282

20. He C, Zheng Z, Zhang J, Li G (2017) Controlled release of natural antibacterial drug loaded by silk biomaterials. Text Bioeng Inform Symp Proc 10:77-90

Publisher's Note Springer Nature remains neutral with regard to jurisdictional claims in published maps and institutional affiliations. 